\title{
Clinical characteristics and outcomes of cancer patients with post-chemotherapy arthritis: a retrospective case series report
}

This article was published in the following Dove Press journal:

Open Access Rheumatology: Research and Reviews

31 May 2017

Number of times this article has been viewed

\author{
Hani Almoallim ${ }^{1-3}$ \\ Sultana Abdulaziz ${ }^{4}$ \\ Eilaf Fallatah' \\ Haya Alhazmi' \\ Nuha Meraiani² \\ Tuqa Bazuhair ${ }^{5}$ \\ Mohammed Mansour \\ Anan Tayeb' \\ Omar Fathaldin ${ }^{6}$ \\ 'Department of Medicine, Faculty of \\ Medicine, Umm Al-Qura University, \\ Makkah, ${ }^{2}$ Department of Medicine, \\ King Faisal Specialist Hospital, \\ Jeddah, ${ }^{3}$ Alzaidi Chair of Research in \\ Rheumatic Diseases, Umm Al-Qura \\ University, Makkah, ${ }^{4}$ Rheumatology \\ Unit, Department of Medicine, King \\ Fahad Hospital, Jeddah, ${ }^{5}$ Department \\ of Medicine, King Abdullah Medical \\ City, Makkah, ${ }^{6}$ Department of \\ Medicine, Faculty of Medicine, \\ King Abdulaziz University, Jeddah, \\ Saudi Arabia
}

Correspondence: Hani Almoallim Department of Medicine, King Faisal Specialist Hospital, PO Box I82I, Jeddah 2I44I, Saudi Arabia

Tel +966505703935

Email hmmoallim@uqu.edu.sa
Objective: The objective of this report was to describe the demographics, clinical characteristics and outcomes of patients with cancer presenting with arthritis following chemotherapy in Jeddah, Saudi Arabia.

Patients and methods: This is a retrospective case series report. We included any patient $\geq 18$ years of age with an established diagnosis of cancer who had received standard therapeutic intervention and was subsequently diagnosed with arthritis after developing rheumatic symptoms either during or after treatment. Patients with clinical evidence of arthritis at the time of their cancer diagnosis were excluded.

Results: Seven cases from different centers were identified. Breast cancer was the most common type of cancer reported. The diagnosis of arthritis was established by a rheumatologist. Bilateral involvement of the metacarpophalangeal and proximal interphalangeal joints was the most common presentation. The knee, back, shoulder and wrist joints were less affected. Following treatment, one patient experienced complete resolution of symptoms, four patients symptomatically improved and one patient had no improvement.

Conclusion: Arthritis can develop both during and after treatment of a malignancy. Solid tumors seem to be more commonly associated with this phenomenon. In this case series, the prognosis was poor as the majority of patients developed persistent arthritis.

Keywords: arthralgia, rheumatic diseases, rheumatoid arthritis, rheumatism, malignancies, breast cancer, autoimmunity

\section{Introduction}

There is a complex association between malignancies and the development of autoimmune and rheumatic diseases. ${ }^{1}$ Malignancies can lead to musculoskeletal disorders as a result of a direct invasion of bones and joints, the development of paraneoplastic syndromes or the generation of autoantibodies. ${ }^{2}$ However, in addition to the malignancies per se, the use of cancer chemotherapeutic agents can also lead to manifestations of rheumatologic diseases. ${ }^{2}$ Although chemotherapeutic agents are an important part of cancer management, they are also associated with a high incidence of adverse effects. ${ }^{3,4}$ The most commonly reported side effects include neuropathy, weight gain, sexual dysfunction, ovarian failure, cardiac toxicity, osteoporosis and secondary hematologic malignancy. ${ }^{4}$ In addition to these well-documented side effects, rheumatologic symptoms have also been reported post chemotherapy. In 1993, Loprinzi et al first reported eight patients with breast cancer who developed post-chemotherapy rheumatism following a standard cyclophosphamide/fluorouracil therapeutic regimen. ${ }^{5}$ 
Subsequently, several studies have described the development of rheumatologic symptoms in different groups of patients, including those with lung cancer, ovarian cancer and Hodgkin's lymphoma as well as patients with hematologic malignancies. ${ }^{6}$ Post-chemotherapy rheumatism may present as rheumatoid arthritis, reactive arthritis, vasculitis or arthritis, with the presence of rheumatoid factor (RF) and antinuclear antibodies (ANAs). ${ }^{5}$

As the number of cancer survivors is increasing globally, a number of factors related to the development of post-chemotherapy rheumatism need to be elucidated. In addition to understanding the epidemiology of this condition, information is also needed regarding the time course for the development of symptoms, the duration of symptoms and whether they resolve over time, along with an assessment of available treatment modalities for this type of arthropathy. Furthermore, increasing physician awareness of post-chemotherapy rheumatism could limit extensive investigations aimed at diagnosing rheumatologic diseases or identifying a recurrence of cancer. ${ }^{7}$ Finally, proper counseling for patients about this adverse effect would undoubtedly lessen their anxiety. ${ }^{5}$

Despite these advantages, there is a lack of studies assessing the prevalence or incidence of chemotherapy-induced arthropathy in cancer patients. Furthermore, because earlier reports restricted their analysis to a subset of patients with breast, ovarian or lung cancer, there is limited information about the risk of rheumatism following treatment with the wide spectrum of cancer chemotherapeutic agents currently available. We compiled this case series from several hospitals in Saudi Arabia to ascertain the demographic profile and clinical outcomes of patients presenting with post-chemotherapy rheumatism. We also sought to identify the most commonly associated malignancies and to investigate if specific cancer chemotherapeutic agents or groups of drugs were more likely to be associated with the development of arthropathy.

\section{Patients and methods}

We conducted a retrospective analysis of all patients who developed arthritis after receiving chemotherapy and who had visited the rheumatology clinic in the following centers in Jeddah, Saudi Arabia: King Faisal Specialist Hospital (KFSH), Dr. Soliman Fakeeh Hospital (DFH) and King Fahad Hospital (KFH), from 1993 to 2015. The research and ethics committee (REC) of KFSH, the REC of the Ministry of Health located at $\mathrm{KFH}$ and the institutional review board of DFH all approved the research proposal. Written consent were obtained from patients or from the next of kin for deceased patients to have the case details published.
We included patients $\geq 18$ years of age with the following characteristics: an established diagnosis of cancer (a solid tumor or a hematological malignancy) based on standard classification and diagnostic procedures; recipients of a standard therapeutic intervention for cancer i.e., surgical, pharmacological (chemotherapy) and nonpharmacological interventions and a confirmed clinical diagnosis of arthritis by a rheumatologist with a history of onset of symptoms either during or after cancer treatment. Patients were excluded if they had documented rheumatologic disease prior to their diagnosis and/or had evidence of direct invasion of their joints resulting in symptoms that could be ascribed to the behavior of the primary tumor.

A data collection sheet was used to collect patient demographics such as age, sex, underlying tumor, chemotherapeutic agents used and other treatments such as radiotherapy and/or surgery if provided. Details regarding the presentation of arthritis were also documented, e.g., number and sites of involved joints, time gap between administration of chemotherapy and the onset of symptoms, severity of the disease and associated symptoms such as swelling and stiffness. Radiologic images and results of the initial laboratory tests were also collected, including C-reactive protein (CRP), erythrocyte sedimentation rate (ESR), ANAs, RF, human leukocyte antigen B27 (HLA-B27) and anti-cyclic citrullinated peptide (ACPA). Any change in the severity of joint symptoms, including complete resolution after initiation of medications, was also documented.

\section{Results}

We identified a total of seven cases in our retrospective analysis. The cases are summarized in Table 1.

\section{Case I}

A 79-year-old male, with known renal insufficiency and hyperuricemia, was referred to KFSH for further evaluation and management of possible multiple myeloma. Before the referral, a skull X-ray revealed the presence of osteolytic lesions, and a bone scintigraphy showed abnormal uptake in the right scapula and left eighth rib. In May 2010, a bone biopsy at KFSH showed mildly hypocellular marrow with moderately increased plasma cell aggregates suggestive of plasma cell myeloma. At that time, the patient was taking painkillers. After 2 months, the patient was prescribed melphalan, dexamethasone and allopurinol. After an additional 3 months, the patient presented to the rheumatology clinic complaining of severe pain in all joints, including both knees, metacarpophalangeal (MCP) joints, wrists and shoulders 


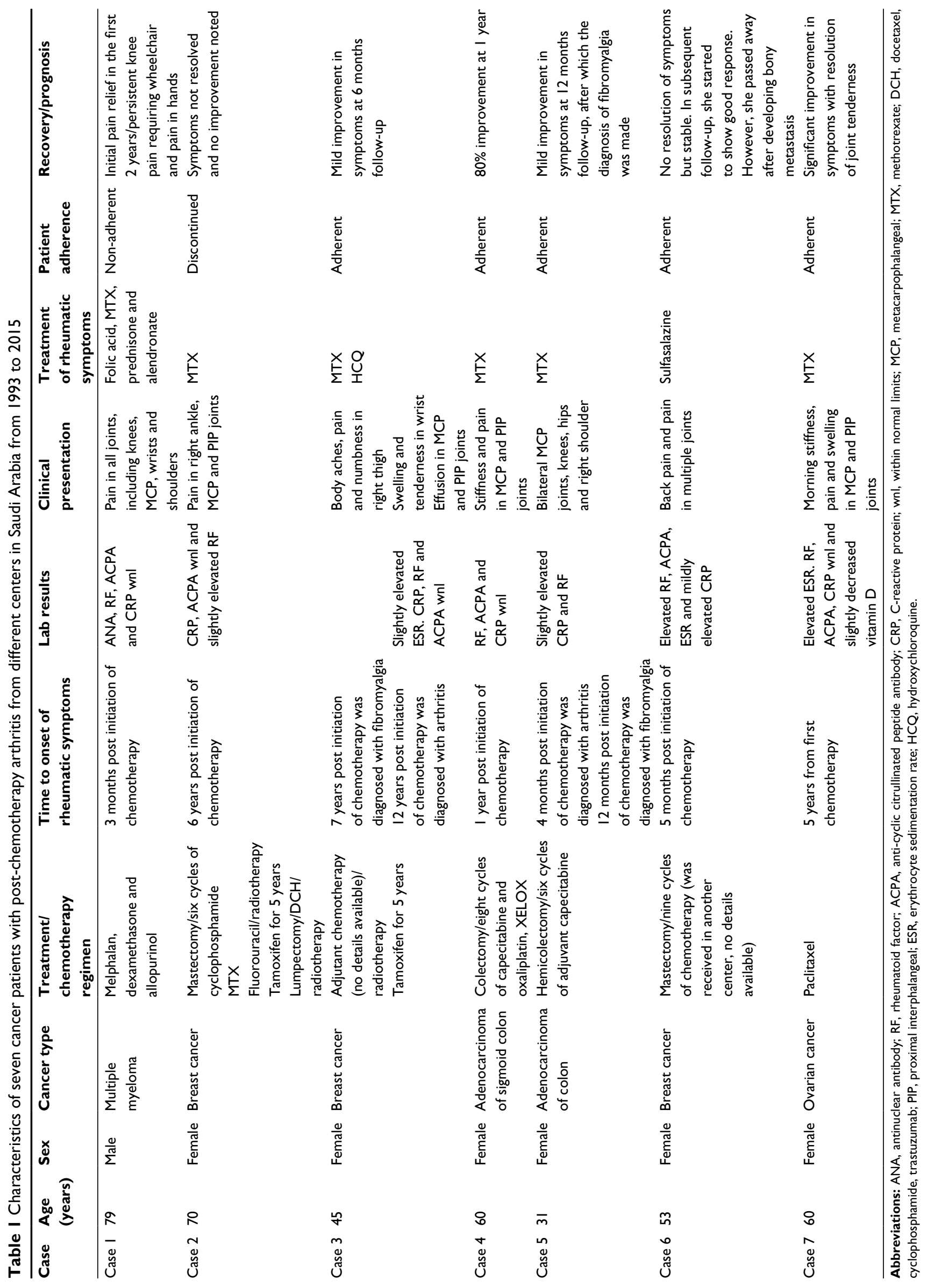


with concomitant loss of function. The pain was severe enough that it interfered with the patient's sleep at night. On examination, there was tenderness in the shoulders, elbows and wrists. All the MCP joints as well as the second, third and fourth proximal interphalangeal (PIP) joints were tender bilaterally. The left knee was also tender, and the right knee was swollen. Under aseptic technique, $27 \mathrm{cc}$ of turbid yellowish synovial fluid was aspirated from the right knee. The fluid analysis revealed high levels of red blood cells (RBCs) and white blood cells (WBCs), but no crystals were visible. The fluid culture and gram stain were negative. Laboratory investigations revealed negative ANA, RF, immunoglobulin $\mathrm{M}(\mathrm{IgM})$ of $5.1 \mathrm{IU} / \mathrm{mL}$ (reference range [RR]: 0-14 IU/mL), ACPA of $\leq 0.5 \mathrm{U} / \mathrm{mL}$ (RR: 0-4.9 U/mL) and a CRP level of $3.73 \mathrm{mg} / \mathrm{L}$ (RR: $0-5.5 \mathrm{mg} / \mathrm{L}$ ). Folic acid was prescribed in addition to methotrexate (MTX) and prednisone, which resulted in pain relief for the following 2 years. However, on the last visit in December 2013, persistent knee pain had required the patient to use a wheelchair. He was also still complaining of pain in the joints of both hands. The patient admitted to being non-adherent to his medication regimen that included prednisone and alendronate at that time.

\section{Case 2}

A 70-year-old female presented to $\mathrm{KFH}$ where she was diagnosed with right breast cancer. She underwent a right mastectomy, followed by six cycles of chemotherapy (cyclophosphamide, MTX and fluorouracil [CMF]) and radiotherapy. She took tamoxifen for the next 5 years. In 2009, the patient was found to have metastasis to her left breast. She underwent a lumpectomy, followed by chemotherapy (docetaxel, cyclophosphamide, trastuzumab [DCH]) and radiotherapy. She continued trastuzumab for 1 year. In 2010, 1 year following her last surgery, she was referred to the rheumatology clinic for complaints of pain and tenderness in her right ankle, MCP and PIP joints bilaterally. On physical examination, there was no swelling, redness or warmth detected near the joints. Laboratory investigations revealed a normal CRP of $2.15 \mathrm{mg} / \mathrm{dL}$ (RR: 0-5.5 mg/L), ACPA of 0.8 U/mL (RR: 0-4.9 U/mL) and a slightly elevated RF of $18.8 \mathrm{IU} / \mathrm{mL}$ (RR: 0-14 IU/mL). The patient was diagnosed with chemotherapy-induced arthritis and was started on MTX $12.5 \mathrm{mg}$ weekly, which was increased to $15 \mathrm{mg}$. After 7 months, she refused to continue the prescribed treatment. Her arthritis persisted.

\section{Case 3}

A 45-year-old female was diagnosed with breast cancer in 2000 , for which she underwent conservative surgery, followed by adjuvant chemotherapy and radiotherapy. Subsequently, she was treated with tamoxifen for 5 years. In 2007, the patient was referred to the rheumatology clinic at KFSH for complaints of body aches accompanied by pain and numbness in her right thigh. She was diagnosed with fibromyalgia. In 2012, she complained of swelling and tenderness in her wrist joint. Laboratory tests revealed ESR of $25 \mathrm{~mm} / \mathrm{h}$ (RR for female 19-50 years: $\leq 20)$, CRP of $8.91 \mathrm{mg} / \mathrm{dL}(\mathrm{RR}$ : 0-5.5 mg/L), RF of $11.4 \mathrm{IU} / \mathrm{mL}$ (RR: 0-14 IU/mL) and ACPA of $<1.0 \mathrm{U} / \mathrm{mL}$ (RR: 0-4.9 U/mL). Ultrasonography (US) showed an effusion in the wrist and first MCP and PIP joints. Based on the abovementioned results, a diagnosis of chemotherapy-induced arthritis was made. The patient was advised to take MTX and hydroxychloroquine (HCQ). At the 6-month follow-up visit, the patient reported mild improvement in her symptoms. She continued on MTX for 4 years, after which time it was discontinued, when her symptoms appeared to be more related to fibromyalgia.

\section{Case 4}

A 60-year-old male underwent sigmoid colectomy for cancer of the sigmoid colon (moderate differentiated adenocarcinoma) diagnosed in 2011. Following surgery, the patient received eight cycles of adjuvant chemotherapy (capecitabine and oxaliplatin, XELOX). In 2012, he complained of pain and swelling in multiple joints with morning stiffness in his MCP and PIP joints, and was referred to the rheumatology clinic at $\mathrm{KFSH}$ for evaluation. Laboratory investigations showed a normal RF of $<10.0 \mathrm{IU} / \mathrm{mL}$ (RR: 0-14 IU/mL), ACPA of $<1.0 \mathrm{U} / \mathrm{mL}$ (RR: 0-4.9 U/mL) and CRP of $2.29 \mathrm{mg} / \mathrm{dL}$ (RR: $0-5.5 \mathrm{mg} / \mathrm{L})$. US showed effusion in the right first and third MCP joints and left fourth MCP joints. The patient was diagnosed with chemotherapy-induced arthritis and was advised to begin MTX $10 \mathrm{mg}$ weekly, which was then increased to $15 \mathrm{mg}$. He was adherent to treatment. In 2013, the patient reported an $80 \%$ improvement in his symptoms.

\section{Case 5}

A 31-year-old female was admitted to KFSH with a 6-month history of abdominal pain that had worsened over the past 2 months. The pain was associated with vomiting, decreased appetite and weight loss. There was no history of lower gastrointestinal (GI) bleeding or change in bowel habits. Surgical history was significant for an appendectomy and a caesarian section. Computed tomography (CT) of the abdomen revealed a $4.5 \times 5.1 \times 5 \mathrm{~cm}$ mass in the mid-ascending colon with involvement of the mesenteric lymph nodes. Colonoscopy showed a second mass, extending an additional 
$10 \mathrm{~cm}$ out from the primary colon mass. Biopsy results confirmed invasive adenocarcinoma. A right hemicolectomy was performed in June 2013. After 2 months, the patient received chemotherapy and completed six cycles of adjuvant capecitabine. Repeated CT of the abdomen showed no evidence of new masses. In February 2014, 4 months after the start of chemotherapy, she developed symptoms of arthritis in her MCP joints, wrists, knees and hips bilaterally as well as in her right shoulder. Physical examination revealed tenderness over her MCP joints, PIP joints, right wrist and left hip joints and decreased range of motion of her interior rotator in her shoulders bilaterally. Laboratory investigations revealed elevated CRP and RF levels. She was treated with MTX for a period of 12 months. Then, MTX was discontinued, and she was diagnosed with fibromyalgia.

\section{Case 6}

A 53-year-old female presented to the breast clinic at KFH in February 2008 complaining of a sudden appearance of a lump in her left breast. A tru-cut needle biopsy revealed grade II breast carcinoma. After 5 months, she underwent a left mastectomy, followed by nine cycles of chemotherapy (chemotherapy was given in another center, and no details are available to us). In January $2009, \sim 5$ months following the mastectomy and the initiation of chemotherapy, the patient was evaluated in the rheumatology clinic for complaints of back pain and pain in multiple joints of 2 months' duration. Laboratory investigations revealed an $\mathrm{RF}$ of $>556 \mathrm{IU} / \mathrm{mL}$ (RR: 0-14 IU/mL), ANA of $4.2 \mathrm{IU} / \mathrm{mL}$ (RR: positive if >1.2), ACPA of $8.7 \mathrm{U} / \mathrm{mL}$ (RR: $0-4.9 \mathrm{U} / \mathrm{mL}$ ), anti-doublestranded DNA antibodies of $5.5 \mathrm{IU} / \mathrm{mL}$ (RR: positive if $>30 \mathrm{IU} / \mathrm{mL}$ ), ESR of $28 \mathrm{~mm} / \mathrm{h}$ (RR: $1-15 \mathrm{~mm} / \mathrm{h}$ ) and CRP of $8.93 \mathrm{mg} / \mathrm{L}$ (RR: $0.02-8 \mathrm{mg} / \mathrm{L}$ ). The patient was diagnosed with rheumatoid arthritis and was prescribed sulfasalazine. At her follow-up visit in August 2010, her symptoms were not resolved, but her condition was stable. In subsequent visits, the patient reported a good response to sulfasalazine. However, she developed bony metastases and passed away.

\section{Case 7}

A 60-year-old female was diagnosed with papillary thyroid cancer in September 2013 at DFH. She received two cycles of radioactive iodine $100 \mu \mathrm{Ci}$ in December 2013 and March 2014. Before 4 years, the patient had been diagnosed and treated for ovarian cancer with cycles of paclitaxel. In February 2014 , she started to complain of morning stiffness, joint pain and swelling in her right third MCP, left second MCP and right third PIP joints. US of the affected joints was consistent with synovitis. Laboratory investigations showed an ESR of $63 \mathrm{~mm} / \mathrm{h}$ (RR: $0-20 \mathrm{~mm} / \mathrm{h}$ ) and a CRP of $8 \mathrm{mg} / \mathrm{dL}$ (RR: $0.02-8 \mathrm{mg} / \mathrm{dL}$ ). RF and ACPA were negative. The vitamin $\mathrm{D}$ level was slightly decreased, $70 \mathrm{nmol} / \mathrm{L}$ (RR: 75-250 nmol/L). The patient was diagnosed with chemotherapy-induced arthritis and advised to begin MTX daily. In April 2014, 4 months after the start of treatment, she noted a significant improvement in her symptoms, with complete resolution of join tenderness. A repeat CRP was unchanged at $8 \mathrm{mg} / \mathrm{dL}$ (RR: $0.02-8 \mathrm{mg} / \mathrm{dL}$ ), and the ESR had decreased to $50 \mathrm{~mm} / \mathrm{h}$ from $63 \mathrm{~mm} / \mathrm{h}$ (RR: $0-20 \mathrm{~mm} / \mathrm{h}$ ).

\section{Discussion}

This case series supports the association between the development of arthritis and treatment with cancer chemotherapeutic agents. We retrospectively identified five female and two male patients from three hospitals who developed rheumatic symptoms after undergoing a chemotherapeutic regimen between 1993 and 2015. It is noteworthy that there were no cases of hematologic malignancies. Furthermore, three of the seven cases were associated with breast cancer. However, because of the small number of patients, it is unclear whether patients with certain types of cancer were more likely to develop postchemotherapy rheumatic disease. It is also interesting that the clinical examination and laboratory investigations were only useful in making the diagnosis of rheumatoid arthritis in two of the seven cases.

The first published report of arthritis post chemotherapy by Loprinzi et al suggested that symptoms generally occur within 1-3 months following the initiation of chemotherapy. Subsequent studies have also reported a short period of time ( $\sim 3-8$ months) before the onset of symptoms. ${ }^{6,8}$ However, the patients in our series presented with symptoms of arthralgia at widely varying time points after the initiation of chemotherapy.

Several studies have sought to establish an association between specific chemotherapeutic agents and the development of rheumatic symptoms. Loprinzi et $\mathrm{al}^{5}$ identified cyclophosphamide/fluorouracil adjuvant therapy as the most common regime associated with post-chemotherapy rheumatism. Standard adjuvant chemotherapy for patients with lung cancer and a combined regimen of cyclophosphamide and tamoxifen among breast cancer patients have been reported to be the most common regimens associated with the development of rheumatic symptoms. ${ }^{6,8}$ Another retrospective analysis that included a mixed group of cancer patients with posttreatment rheumatism found that fluorouracil was the most commonly prescribed drug, followed by 
cyclophosphamide and cisplatin. ${ }^{9}$ However, in our series, there was no single agent or group of drugs more prominently associated with post-chemotherapy arthritis.

There is also variability in the symptomatic presentation of patients with post-chemotherapy rheumatic disease. Among our cases, the MCP, PIP and knee joints were the most commonly involved anatomic locations, whereas other studies have reported the most commonly involved joints to be the toes, shoulders, fingers, ankles and knees. ${ }^{6,9,10}$

In our series, two patients underwent long-term treatment with tamoxifen, an aromatase inhibitor, which is well known to cause arthralgia. ${ }^{4,10}$ The acute decrease in estrogen production by aromatase inhibitors is thought to be responsible for most of the symptoms. ${ }^{11}$ Symptoms typically develop within 2-6 months of the initiation of tamoxifen therapy and generally resolve over time without any evidence of structural damage of the affected joints. ${ }^{11}$ In contrast to earlier reports, our patients developed symptoms long after the start of tamoxifen therapy, although there was also improvement with treatment.

There is no consensus regarding the optimal therapeutic management of post-chemotherapy rheumatic disease. Earlier studies $5,6,8,9,11$ have reported variable response rates to treatment with non-steroidal anti-inflammatory drugs or disease-modifying antirheumatic drugs. In our series, none of our patients were prescribed NSAIDs. The patients received MTX, HCQ, sulfasalazine or prednisolone. Four patients experienced symptomatic improvement following treatment with MTX and sulfasalazine.

Although the limitations of this report include its retrospective nature and the small number of patients, its unique perspective is the inclusion of patients from three different hospitals with a diversity of malignancies.

\section{Conclusion}

The presentation of chemotherapy-associated rheumatic disease varies among patients in terms of the timing of presentation, the types of symptoms and the prognosis following treatment. Increased awareness of this adverse side effect is likely to have several benefits. The number of unnecessary investigations by health care providers would potentially decrease. In addition, educating patients about the possibility of developing rheumatic symptoms following chemotherapy would likely lower their level of anxiety. Finally, increased reporting of the occurrence of this side effect associated with chemotherapy will help to better determine its true incidence and prevalence.

\section{Acknowledgments}

The report was funded by Alzaidi Chair of Research in Rheumatic Diseases, Umm Al-Qura University. The authors would like to acknowledge Dr Hanan Alosaimi for her help during the manuscript preparation and Dr Somaia Alsafri for her contribution and help in the data collection.

\section{Disclosure}

The authors report no conflicts of interests in this work.

\section{References}

1. Abu-Shakra M, Buskila D, Ehrenfeld M, Conrad K, Shoenfeld Y. Cancer and autoimmunity: autoimmune and rheumatic features in patients with malignancies. Ann Rheum Dis. 2001;60(5):433-441.

2. Alias A, Rodriguez EJ, Bateman HE, Sterrett AG, Valeriano-Marcet J. Rheumatology and oncology: an updated review of rheumatic manifestations of malignancy and anti-neoplastictherapy. Bull NYU Hosp Jt Dis. 2012;70(2):109-114.

3. Wright AA, Zhang B, Keating NL, Weeks JC, Prigerson HG. Associations between palliative chemotherapy and adult cancer patients' end of life care and place of death: prospective cohort study. BMJ. 2014;348:g1219.

4. Creamer P, Lim K, George E, Dieppe P. Acute inflammatory polyarthritis in association with tamoxifen. Br J Rheumatol. 1994;33(6):583-585.

5. Loprinzi CL, Duffy J, Ingle JN. Postchemotherapy rheumatism. J Clin Oncol. 1993;11(4):768-770.

6. Amiri AH, Jaferian S. Post-chemotherapy arthralgia and arthritis in lung cancer. South Asian J Cancer. 2012;1(2):72-75.

7. The UPPSALA MONITORING CENTRE. WHO Causality Assessment Scale. 2016. Available from: http://who-umc.org/Graphics/24734.pdf. Accessed September 13, 2016.

8. Amiri AH, Rafiei A. Analysis of patients with post-chemotherapy arthralgia and arthritis in breast cancer. Indian J Med Sci. 2010;64(5):197-203.

9. Kim MJ, Ye YM, Park HS, Suh CH. Chemotherapy-related arthropathy. J Rheumatol. 2006;33(7):1364-1368.

10. Burstein HJ. Aromatase inhibitor-associated arthralgia syndrome. Breast. 2007;16(3):223-234.

11. Younus J, Kligman L. Management of aromatase inhibitor-induced arthralgia. Curr Oncol. 2010;17(1):87-90.
Open Access Rheumatology: Research and Reviews

\section{Publish your work in this journal}

Open Access Rheumatology: Research and Reviews is an international, peerreviewed, open access journal publishing original research, reports, editorials, reviews and commentaries on all aspects of clinical and experimental rheumatology in the clinic and laboratory including the following topics: Pathology, pathophysiology of rheumatological diseases; Investigation, treatment and

\section{Dovepress}

management of rheumatological diseases; Clinical trials and novel pharmacological approaches for the treatment of rheumatological disorders. The manuscript management system is completely online and includes a very quick and fair peer-review system, which is all easy to use. Visit http://www.dovepress.com/ testimonials.php to read real quotes from published authors. 